\title{
A ROS IMPLEMENTATION OF THE MONO-SLAM ALGORITHM
}

\author{
Ludovico Russo, Stefano Rosa, Basilio Bona ${ }^{1}$ and Matteo Matteucci ${ }^{2}$ \\ ${ }^{1}$ Dipartimento di Automatica e Informatica, Politecnico di Torino, Torino, Italy \\ \{ludovico.russo, stefano.rosa, basilio.bona\} @polito.it \\ ${ }^{2}$ Dipartimento di Elettronica, Informatica e Bioingegneria, \\ Politecnico di Milano, Milano, Italy \\ matteo.matteucci@polimi.it
}

\begin{abstract}
Computer vision approaches are increasingly used in mobile robotic systems, since they allow to obtain a very good representation of the environment by using low-power and cheap sensors. In particular it has been shown that they can compete with standard solutions based on laser range scanners when dealing with the problem of simultaneous localization and mapping (SLAM), where the robot has to explore an unknown environment while building a map of it and localizing in the same map. We present a package for simultaneous localization and mapping in ROS (Robot Operating System) using a monocular camera sensor only. Experimental results in real scenarios as well as on standard datasets show that the algorithm is able to track the trajectory of the robot and build a consistent map of small environments, while running in near real-time on a standard $P C$.
\end{abstract}

\section{KEYWORDS}

SLAM, Mono-SLAM, Mapping, Mobile robotics

\section{INTRODUCTION}

In several application scenarios mobile robots are deployed in an unknown environment and they are required to build a model (map) of the surroundings, as well as localizing therein.

Simultaneous localization and mapping (SLAM) applications now exist in a variety of domains including indoor, outdoor, aerial and underwater and using different types of sensors such as laser range finders, sonars and cameras [4]. Although, the majority of those approaches still rely on classical laser range finders, the use of vision sensors provides several unique advantages: they are usually inexpensive, low-power, compact and are able to capture higher level infor- mation compared to classical distance sensors. Moreover, human-like visual sensing and the potential availability of higher level semantics in an image make them well suited for augmented reality applications.

Visual SLAM approaches are usually divided in two main branches: smoothing approaches based on bundle adjustment, and filtering approaches based on probabilistic filters. The latter are divided in three main classes: dense, sparse and semantic approaches. Dense approaches ([17], [14], [22]) are able to build dense maps of the environment, which make the algorithms more robust but at the same time heavy in terms of computational requirements; indeed, most of these

David C. Wyld et al. (Eds) : CST, ITCS, JSE, SIP, ARIA, DMS - 2014

pp. 339-351, 2014. (C) CS \& IT-CSCP 2014

DOI : $10.5121 /$ csit.2014.4131 
approaches are able to work in real-time only when dedicated hardware (e.g., a GPU or FPGA) is used. Sparse approaches ([15], [7], [12]) address the problem of computational requirements by sparsifying the map; obviously this choice impacts on the robustness of the solution. These algorithms require less computational efforts because they try to allocate in memory only the most significant key points representing the map; for this reason, they are natural candidates for a real time Visual SLAM implementation. Semantic approaches ([10],[20]), extract higher level semantic information from the environment in order to build a more robust and compact map.

Parallel Tracking and Mapping (PTAM) was proposed in [15] as a sparse approach based on a monocular camera targeted to augmented reality applications. The main idea of PTAM is to divide tracking and map updating phases. Camera pose tracking is performed at each time step, by comparing the new frame with the current map using feature matching techniques; FAST features [18] are used for match- ing. Map updating is performed only on a set of key frames and when the current camera position estimation is precise enough. The algorithm requires an initialization phase in which the same features are viewed from different points of view. The most important limitation of the algorithm is the impossibility to handle occlusions.

A promising solution is the Mono-SLAM algorithm, originally proposed by Davison et al. in [12]. In this approach, the map and the camera pose are stored as stochastic variables and the system evolution is estimated by an incremental Extended Kalman Filter (EKF). Inverse depth parametrization can be used for the representation of point features, which permits efficient and accurate representation of uncertainties [16]. By using an approach is known as active search paradigm [11], the algorithm is able to speed-up feature matching, since interesting points in the each new frame are looked for only in the most probable regions. In addition, the algorithm does not need an initialization phase and its probabilistic nature makes it more robust to occlusions. The most evident limitation of the algorithm is the fact that, when the map becomes too large, the EKF processing phase becomes too computationally heavy to be computed in real- time [21]. However, some solutions have been proposed to solve that problem too [13].

In this work we present an implementation of the Mono-SLAM algorithm using the ROS [3] framework. The developed ROS node takes as input the images captured from a monocular camera and outputs the trajectory of the camera, as well as a point map representing the environment around the robot. The algorithm is able to run in near real-time on a standard PC with no dedicate hardware for small and medium length trajectories. The rest of the paper is organized as it follows: in Section 2 we briefly recall the formulation of the Mono-SLAM problem; in Section 3 we describe our implementation of the algorithm; in Section 4 we show experimental results that validate the effectiveness of the approach; finally in Section 5 we draw some conclusions and discuss about future extensions of our implementation.

\section{PROBLEM FORMULATION}

As in classical SLAM approaches based on laser scanners, the robot pose is described as a stochastic variable with Gaussian distribution, and the map of the environment is sparse. The environment is described by a limited set of features $\left\{\boldsymbol{f}_{i}\right\}$, i.e., measurable geometrical entities (points in the case of Mono-SLAM). Features are described as gaussian variables, as well.

The system state, identified by the robot pose and the map, is represented at any time $\mathrm{t}=$ $\mathrm{k} \Delta \mathrm{t}$, where $\Delta \mathrm{t}$ is the time elapsed since the previous step, as a stochastic variable with Gaussian distribution 
$\hat{\boldsymbol{\mu}}_{k} \sim \mathrm{N}\left(\boldsymbol{\mu}_{k}, \boldsymbol{\Sigma}_{k}\right)$

having $\boldsymbol{\mu}_{k \text { mean and covariance }} \Sigma k$.

The state vector encapsulates the information on both camera pose and world features

$\boldsymbol{\mu}_{k}=\left(\begin{array}{llll}x_{k}^{T} & \boldsymbol{f}_{1}^{T} & \cdots & \boldsymbol{f}_{m}^{T}\end{array}\right)^{T}$

Information concerning the camera motion at any instant is encoded in the vector built as

$\boldsymbol{x}_{k}=\left(\begin{array}{llll}\boldsymbol{r}_{k}^{T} & \mathrm{q}_{k}^{T} & \boldsymbol{v}_{k}^{T} & \boldsymbol{\omega}_{k}^{T}\end{array}\right)^{T}$

where the vector $\boldsymbol{r}$ and the quaternion q represent the pose of the camera reference frame $\mathrm{C}$

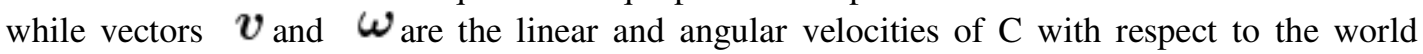
reference frame $\mathrm{W}$.

The function used to predict the evolution of the camera state is given by

$\boldsymbol{x}_{k+1 \mid k}=\boldsymbol{g}_{v}\left(\boldsymbol{x}_{k}\right)=\left(\begin{array}{c}\boldsymbol{r}_{k}+\left(\boldsymbol{v}_{k}+\boldsymbol{V}_{k}\right) \Delta t \\ \mathrm{q} k \times \text { quat }\left(\left(\boldsymbol{\omega}_{k}+\boldsymbol{\Omega}_{k}\right) \Delta t\right) \\ \boldsymbol{v}_{k}+\boldsymbol{V}_{k} \\ \boldsymbol{\omega}_{k}+\boldsymbol{\Omega}_{k}\end{array}\right)$,

Where quat $\left(\left(\boldsymbol{\omega}_{k}+\boldsymbol{\Omega}_{k}\right) \Delta t\right)_{\text {is }}$ the quaternion corresponding to the rotation $\left(\boldsymbol{\omega}_{k}+\boldsymbol{\Omega}_{k}\right) \Delta t$ obtained from the axis-angle representation while $\boldsymbol{V}_{k}^{-}$and $\boldsymbol{\Omega}_{k}$ are the noise vectors affecting respectively linear and angular velocities. Features are consider static so they do not need a prediction model.

The measurements function necessary to perform the update step of the EKF filter is given by

$$
\boldsymbol{h}(\boldsymbol{\mu})=\left(\begin{array}{c}
h\left(\boldsymbol{f}_{1}, \boldsymbol{x}_{k}\right) \\
\vdots \\
h\left(\boldsymbol{f}_{m}, \boldsymbol{x}_{k}\right)
\end{array}\right)
$$

where the projection function $\mathrm{h}()$ is a function able to project a $3 \mathrm{D}$ features in the image plane using the predicted camera pose.

\section{IMPLEMENTATION}

The software has been developed using the Robot Operating System (ROS) [3] in C++ under Linux. The OpenCV library [1] was used for image processing and the Eigen3 library [2] was used for matrix operations. 
The architecture of the implemented solution is described in Figure 1. Each new frame fk is acquired from the camera sensor topic by the capture and preprocess block, which performs some preprocessing and feeds the frame to the Mono-SLAM super-block, which is the main block in charge of processing the frame in order to reconstruct the camera motion and the map. The output of the Mono-SLAM main block at each step is the estimated state of the system, as in equation (2).

The feature matching block is in charge of matching, in each new frame, the predicted features contained in the set $\mathrm{P}_{k \sqcap k-1}^{\prime}$. In order to improve matching klk-1 performances, we implemented an active search technique [11]: candidates for new corresponding features are searched only in the most probable areas (which are modeled using ellipsoids), where there is the $99 \%$ probability of finding them. This block uses information from the predicted measurements and its related covariance $S_{\mathrm{k} \square \mathrm{k}-1}$ in order compute the measurement vector $\boldsymbol{y}_{k}$.

When $\boldsymbol{y}_{k}$ has been computed, the algorithm performs the standard EKF update and prediction steps. The innovation vector $\boldsymbol{e}_{k}=\boldsymbol{y}_{k}-\dot{y}_{k \mid k-1}$ is computed; then the filter performs update and prediction steps in order to estimate, respectively, the updated state $\boldsymbol{\mu}_{k \mid k}$ with its covariance $\boldsymbol{\Sigma}_{k \mid k}$ and the predicted state $\boldsymbol{\mu}_{k+1 \mid k}$ with its covariance $\boldsymbol{\Sigma}_{k+1 \mid k}$. In the proposed implementation, the update step includes the 1-Point RANSAC algorithm [9] for outliers rejection, in order to improve robustness.

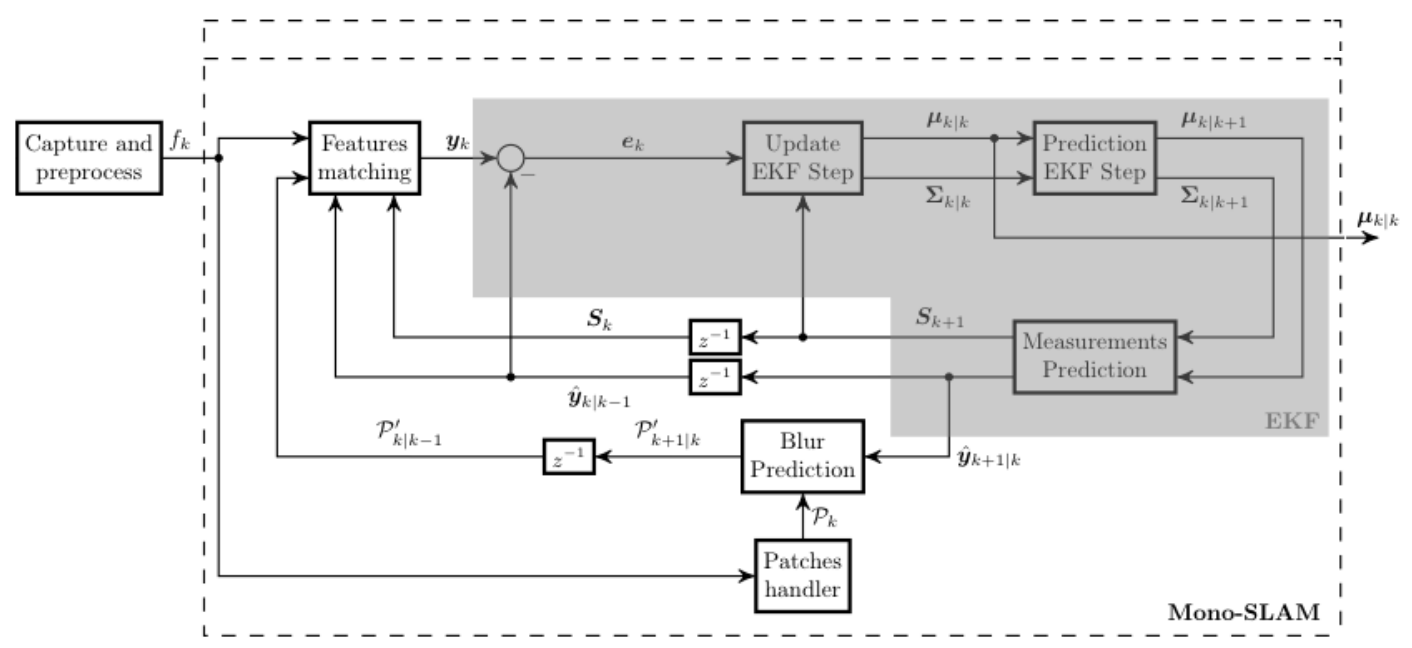

Figure 1. Architecture of the proposed solution.

The patches handler block is in charge of managing the Pk set. In particular, it is used to find the best features to track at the very beginning (i.e., when the first frame is being acquired) and to delete old features that are no more useful (e.g., when they exit from the frame bounds) and must be replaced. More details are reported in Section 3.1.

Blur prediction block is used to predict the motion blur affecting patches when the camera undergoes quick motion. Details are reported in Section 3.2.

Note that the $\mathrm{z}^{-1}$ block represents a delay equal to the inverse of the camera frame rate, which is used to store predicted information until a new frame has been acquired. 


\subsection{Feature matching}

The task of features matching consists in finding correspondences for a set of features when a new frame is acquired. Each feature is associated with a descriptor, which will be discussed later in this subsection.

Once a new image is acquired, new meaningful features are found and their descriptors are computed. Then, correspondences are found between the new features and the features from the previous frame. Matching techniques which are fast enough to be used in real-time are usually not robust enough for finding correct correspondences. Luckily, using a probabilistic approach gives an advantage, since the regions where we look for correspondences can be reduced to the ones where the features are more probable to be found. In other words, the search for matches is performed in a region around the prediction of the measurements and the size of the region is related to the covariance matrix of that features; intuitively, the larger the uncertainty on the position of the features, the wider the searching region should be.

In Mono-SLAM, that region is given by projecting the ellipsoid related to the prediction of the feature fi in the image plane, where $s$ is a number specifying the size of the region, usually $s=2$ or $\mathrm{s}=3$, i.e., $95 \%$ or $99 \%$ of probability of finding the feature. Please note that the size of this region is given by $\mathrm{s} 2 \mathrm{Si}$, where $\mathrm{Si}$ is the $2 \times 2$ submatrix of $\mathrm{Ss}$ related to the measurements estimation of. Hence, the matching is performed on the ellipsoid Si having center in pi and size s2Si mathematically described as follows:

$\mathrm{S}_{i}=\left\{p \in \mathcal{I} \mid\left(p-p_{i}\right)^{T} \mathbf{S}_{i}^{-1}\left(p-p_{i}\right) \leqslant s^{2}\right\}$.

If the feature can be matched inside this search region, then it is considered as successfully matched. The image coordinates zi, denoting the position of the feature in the new frame, are appended to the measurement vector $\boldsymbol{z}_{k}$ and the feature will contribute towards the correction of the estimates mean $\boldsymbol{\mu}_{k}$ and covariance $\Sigma k$. Otherwise the predicted measurement for $\boldsymbol{f}_{i}$ will

be removed from $\boldsymbol{h}_{k}$, since $\boldsymbol{z}_{k}$. there is no corresponding measurement in. Subsequently, the corresponding rows of are deleted from the Jacobian Hk of vector $\boldsymbol{h}_{k}$. This method, known as active search, makes the algorithm to be much more robust and it allows also to use very simple and fast features descriptors to perform matching. For this reason, in this work a simple patch matching techniques was used, as in the original work in [12] .

A patch is defined as a sub image of given dimensions (usually small) extracted from an image around a specific pixel. In our work the center of each patch is found by an interest point detector. Patch matching requires to find the position of the center of the patch inside the original image, i.e., the interest point. The normalized cross correlation operator is the default solution to perform patch matching. Given two patches $\mathrm{P}$ and $\mathrm{P}^{\prime}$ of the same dimensions, the cross correlation score between the two patches is defined as

$\mathrm{C}_{N C C}\left(P, P^{\prime}\right)=\frac{1}{n} \sum_{u} \sum_{v} \frac{(P(u, v)-\bar{P})\left(P^{\prime}(u, v)-\bar{P}^{\prime}\right)}{\sqrt{\sigma_{P}^{2} \sigma_{P^{\prime}}^{2}}}$,

where $\mathrm{n}$ is the number of pixels contained in each patch and $\overline{\mathrm{P}}$ and $\sigma \mathrm{P} 2$ are respectively mean and covariance of the intensity of the pixels of $\mathrm{P}$, defined as 


$$
\begin{aligned}
\bar{P} & =\frac{1}{n} \sum_{u} \sum_{v} P(u, v), \\
\sigma_{P}^{2} & =\frac{1}{n} \sum_{u} \sum_{v}(P(u, v)-\bar{P})^{2}=\frac{1}{n} \sum_{u} \sum_{v} P^{2}(u, v)-\bar{P}^{2} .
\end{aligned}
$$

Patch matching is performed computing $\mathrm{C}_{N C C}$ between the patch to find and each patch having center in the search region $\mathrm{Si}$, defined above. The center of the patch which maximize $\mathrm{C}_{N C C}$ is chosen as best match.

\subsection{Blur correction}

When the camera motion is very pronounced, patch match- ing could fail due to the amount of motion blur affecting the acquired camera frames. To handle motion blur, each patch is preblurred using the speed information contained in the predicted state, and the blurred patches are used to perform matching. Unlike alternative solutions that restore the whole image in order to handle blur, this solution uses the Mono- SLAM paradigm in order to reduce computational efforts, by applying blur prediction only to few patches instead of the whole image. More details on the implementation of this approach are reported in a previous work [19].

\subsection{1-Point RANSAC}

1-Point RANSAC algorithm for EKF filters has been originally proposed in [9]. The algorithm is composed by two parts: the first one is in charge of selecting low-innovation inliers, while the second one selects high-innovation inliers. Low-innovation inliers are selected by executing RANSAC using a single feature (point) to generate hypotheses and select the best consensus set. Unlike classical RANSAC algorithm, the hypotheses are generated using also information given by the EKF filter: new hypotheses are generated by performing EKF update on the selected points only. Then, a consensus set is created by collecting all measured points which lay inside a certain probability ellipsoid (given by a threshold) centered in the predicted measurements obtained from the computed hypothesis.

Low-innovation inliers are elements of the consensus set of the best hypothesis after RANSAC execution. They are assumed to be generated by the true model since they are at a small distance from the most supported hypothesis. The remaining points could be both inliers and outliers, even if they are far from the supported hypothesis. This is due to the fact that the point chosen to generate the best hypothesis could not contain all the information needed to correctly update the state. For instance, it has been explained that distant points are useful for estimating camera rotation, while close points are needed to estimate translation. In the RANSAC hypotheses generation step, a distant feature would generate a highly accurate 1-point hypothesis for rotation, while translation would remain inaccurately estimated. Other distant points would, in this case, have low innovation and would vote for this hypothesis. But as translation is still inaccurately estimated, nearby points would presumably exhibit high innovation even if they are inliers.

High-innovation inliers are selected after a partial EKF update step involving only the lowinnovation inliers selected by RANSAC. After this partial update, most of the correlated error in the EKF prediction is corrected and the covariance is greatly reduced. This high reduction will be exploited for the recovery of high-innovation inliers: as correlations have weakened, consensus for the set will not be necessary to compute and individual compatibility will suffice to discard inliers from outliers. For each point discarded by the RANSAC algorithm, we check if it is individually compatible by verifying whether it lies in a fixed-sized (multiple of its covariance) 
probability region or not. If the check is passed, the point is added to the high-innovation inliers set. After that all points are checked, a second update involving the high-innovation inliers is performed.

\subsection{Inverse depth coding}

In order to tackle the problem of features initialization and features at infinity, in an alternative representation was proposed called inverse depth, which represents each feature with six parameters

$\boldsymbol{\psi}=\left(\begin{array}{llllll}x^{\mathcal{C}} & y^{\mathcal{C}} & z^{\mathcal{C}} & \theta & \phi & \rho\end{array}\right)^{T}$,

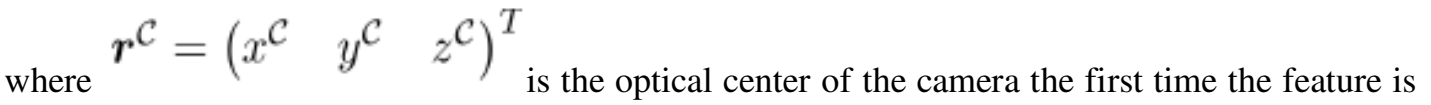
observed, $\theta$ and $\phi$ are respectively azimuth and elevation of the feature with respect to the image coordinate system and $\rho=1 / \mathrm{d}$ is the so-called inverse depth of $\boldsymbol{f}$, where $\mathrm{d}$ is the distance of the feature from the optical center the first time it is observed. The 3D position of the features in $\mathrm{W}$ can be computed as

$$
\begin{aligned}
& \boldsymbol{y}=\boldsymbol{r}^{\mathcal{C}}+\frac{1}{\rho} \boldsymbol{\eta}(\theta, \phi) \\
& \boldsymbol{\eta}(\theta, \phi)=\left(\begin{array}{lll}
\sin \theta \cos \phi & -\sin \phi & \cos \theta \cos \phi
\end{array}\right)^{T}
\end{aligned}
$$

The advantage of using inverse depth encoding is that it allows to compute a normalized vector parallel to $\boldsymbol{y}_{\text {so defined }}$

$$
\hat{\boldsymbol{u}}=\frac{1}{d} \boldsymbol{y}=\rho \boldsymbol{r}^{C}+\boldsymbol{\eta}(\theta, \phi)
$$

which is computable also in cases of features at infinity, i.e., with $\rho \rightarrow 0$. Note that inverse depth features can not represent point with zero depth, because this implies $\rho \rightarrow \infty$. This is not a problem because a features with zero or very small depth implies a real point coincident or very near to the camera optical center, i.e., inside the optics of the camera.

\section{ROS IMPLEMENTATION DETAILS}

The algorithm has been fully implemented in ROS as a node called Mono- SLAM. The node subscribes to an image topic containing the video stream coming from a video camera (in our work the topic is published by camera1394 or gscam ROS nodes). The full source code for our implementation will soon be available online on the repository of our laboratory ${ }^{1}$.

The Mono-SLAM node publishes a set of topics which are the camera pose, the camera trajectory, the reconstructed point-map. Other topics which are useful for debugging purposes are the image frames showing the tracked features as well as their state (new, normal, discarded by RANSAC), and the covariances of all features. The topics can be visualized using the rviz ROS node. Figure 2 shows the different subscribed and published topics.

${ }^{1}$ https://github.com/rrg-polito 
Some examples of the node in action are shown in Figure 3 and 4. Figure 3 shows some frames elaborated by the algorithm. Several information are given:

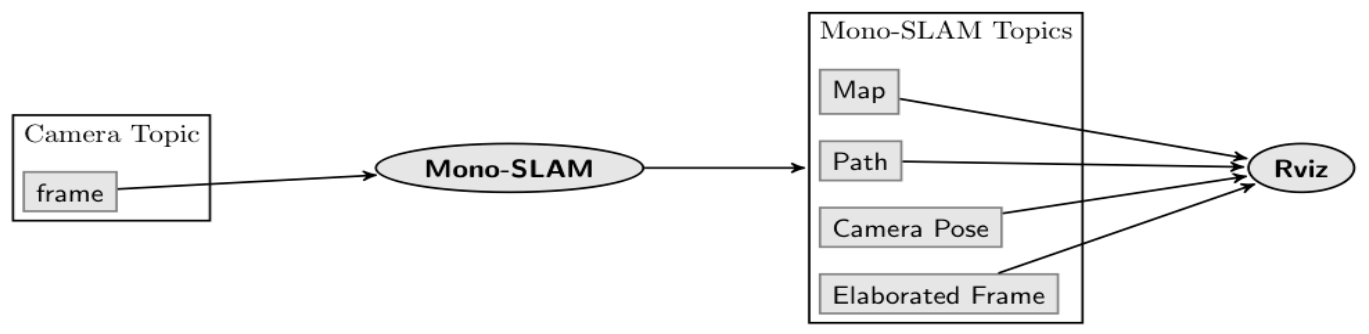

Figure 2. ROS graph of the developed solution.

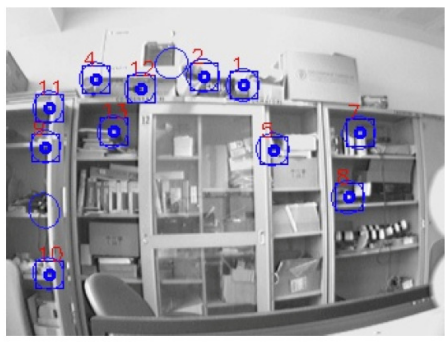

(a)

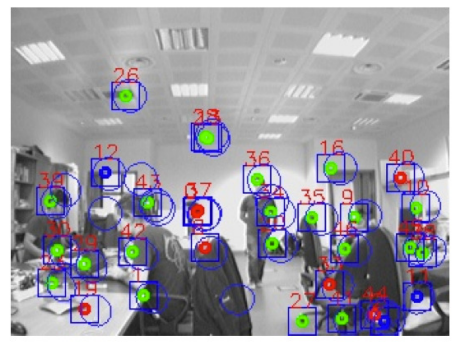

(b)

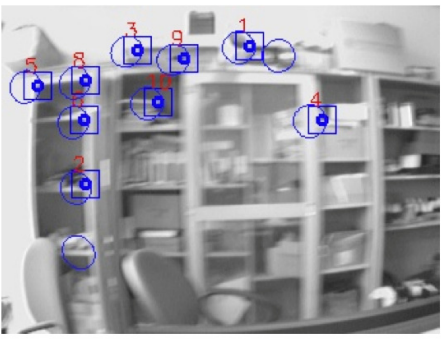

(c)

Figure 3. Examples of the algorithm in action. Positions of the patches are shown in each frame, as well as the ellipsoids that represent their predicted positions in the next frame. (a) shows a standard frame.

(b) shows the 1-Point RANSAC algorithm working: blue patches are low-innovation inliers, green patches are high-innovation inliers and red patches are rejected measurements. (c) shows enhances prediction.

correctly matched patches are shown by blue rectangles, features selected as low- innovation inliers are shown as blue points, features selected as high-innovation inliers are shown as green points, features which are discarded by the 1-point RANSAC algorithm are shown as red points and finally the high probability region in which each feature should lie in the next frame is marked by an ellipse. Moreover, Figure 4 shows some examples of the visual output provided by rviz ROS node. The camera trajectory and the reconstructed map are shown. Each 3D feature is shown together with its covariance ellipsoid. The green covariance refers to features in inverse depth coding while the red ones refer to features in Euclidian representation. Please note that, due to limits of rviz, the covariance of the inverse depth features is represented by projecting the features in the Euclidian space, i.e., always as an ellipsoid, instead of correctly representing that covariance in the inverse depth space, i.e., with a conic shape. 


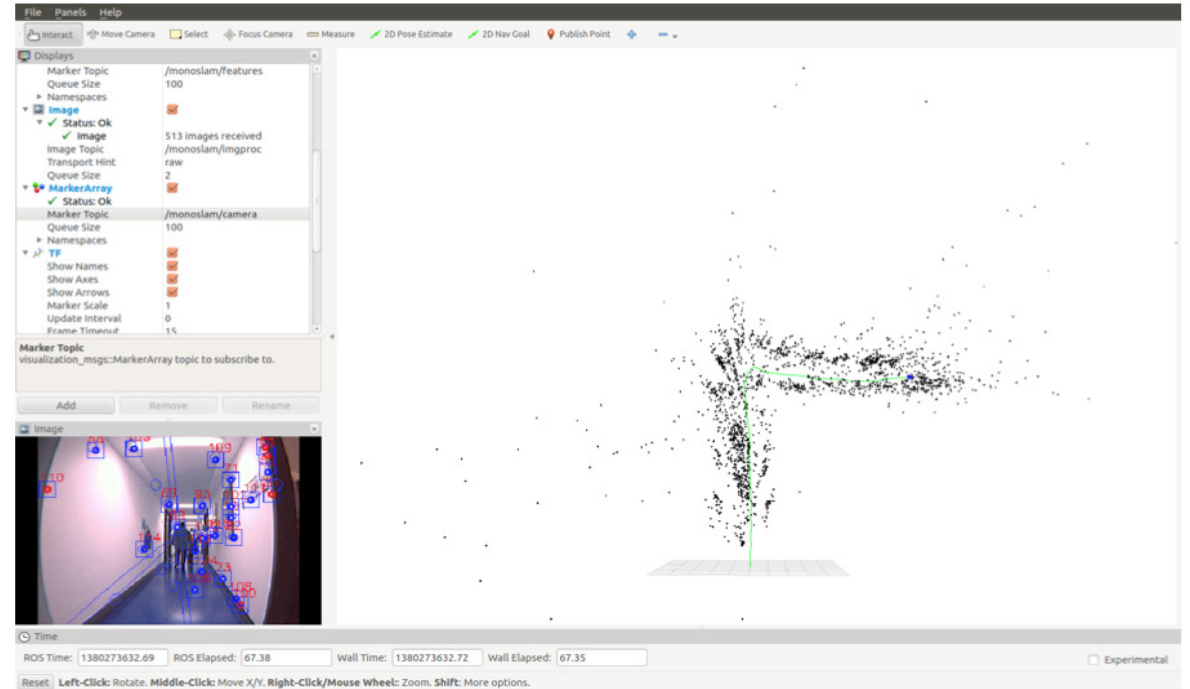

(a)
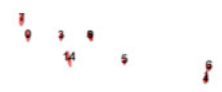

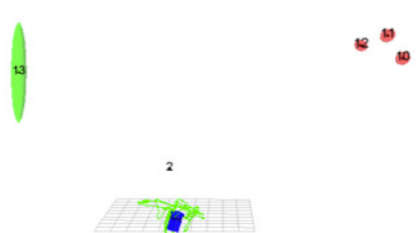

(b)

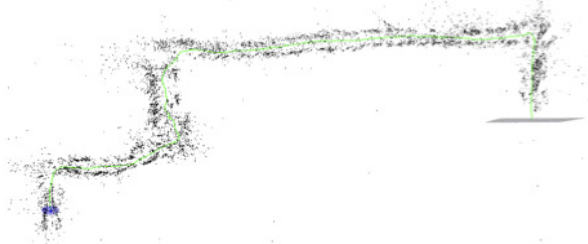

(c)

Figure 4. Output from the algorithm can be visualized using the rviz ROS node. (a) shows a screenshoot of the complete rviz environment: camera image with detected features is shown at the bottom-left, while in the main area the resulting 3D map is shown as well as the camera pose and its trajectory (in green).

(b) shows the visualization of the ellipsoids representing the covariance on the pose of the features in 3D. Green ellipsoids are associated to inverse depth features while red ellipsoids to Euclidian features.

(c) shows a larger map reconstructed by the algorithm.

\section{EXPERIMENTAL RESULTS}

In order to evaluate the proposed algorithm, experimental tests were carried out using a standard monocular camera. Moreover the algorithm has been tested on a standard benchmarking dataset for robotic systems. All the experiments have been carried out on a standard PC equipped with an Intel i7 3.4 GHz CPU and $4 \mathrm{~GB}$ of RAM.

\subsection{Hand held camera}

We first tested the algorithm using a simple handheld FireWire camera. We tested robustness to occlusions by introducing an object (hand) in front of the camera while the algorithm was running. Figure 5 shows that the approach is able to reject occluded points and to match them correctly again when the occluding object is removed from the scene. 


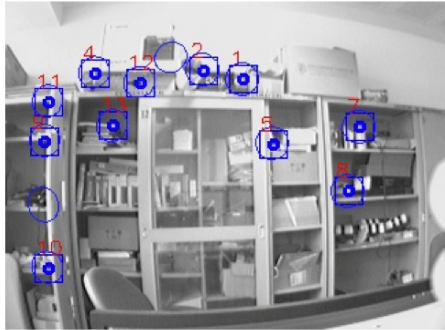

(a)

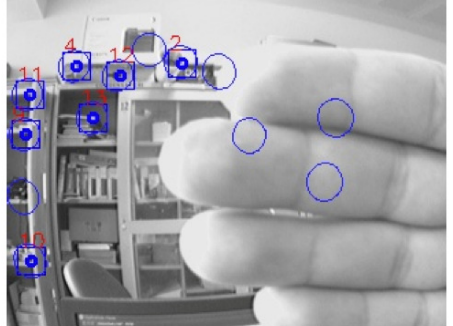

(b)

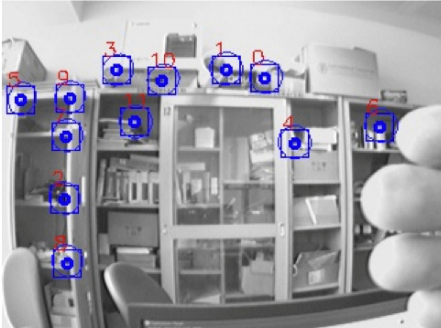

(c)

Figure 5. Testing robustness to occlusions. (a) The algorithm is working nor- mally. (b) Patches occluded by the hand are discarded. (c) The hand is removed from the scene patches are matched again.

In another experiment a moving object (person) is present in the scene. In Figure 6 a person is moving in front of the camera. RANSAC is able to rejects moving features, which are not matched. Occluded features are also discarded.

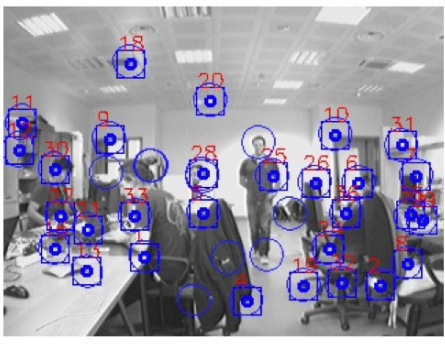

(a)

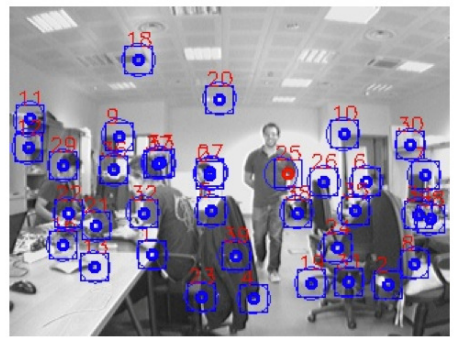

(b)

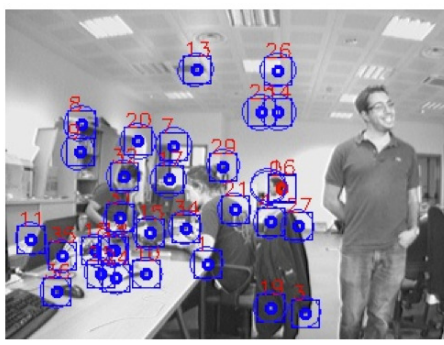

(c)

Figure 6: Testing robustness to moving objects. In (a) the person starts moving. In (b) feature 25 is rejected by RANSAC. In (c) occluded features are removed from the state.

\subsection{Rawseeds dataset}

We also tested our algorithm on the datasets freely available from the Rawseeds Project [5]. These are high-quality multi-sensor datasets, with associated ground truth, of rovers moving in large environments, both indoor and outdoor. For each sensor, calibration data is provided. For the experiments we used the video stream coming from the front camera of the rover in both indoor and outdoor scenarios.

The robot trajectory estimated by our approach was compared with the ground truth available from the datasets. The ground truth is composed by the trajectory obtained from a multi-camera system and visual tags mounted on the robot, which is not available for the whole length of the trajectory, and the estimated trajectory coming from a standard SLAM algorithm based on laser scanner sensors; for the outdoor dataset GPS data is used for the ground truth.

For indoor experiments, we used the Bicocca_2009-02-26a dataset ${ }^{2}$, in which the robot is moving in an indoor dynamic environment. Some results obtained from this dataset are shown in Figure 7. It is possible to note that the algorithm was able to perform with good accuracy on small and medium scales (10 or plus meters), while on large scales the trajectory is far from the real one.

${ }^{2}$ http://www.rawseeds.org/rs/datasets/view/6 
This is due to the fact that the Mono-SLAM algorithm does not explicitly perform loop closing. Loop closing only happens when new patches are correctly matched to previous patches, but this is difficult after large displacements of the camera. Another issue is that the algorithm is not able to correctly measure rotations in some cases. This is due to the lack of significant features in some areas (e.g., when facing a wall) or in presence of too many moving objects in front of the robot.

For outdoor experiments Bovisa_2008-10-04 dataset ${ }^{3}$ has been used. Also this second experiment (see Figure 8) shows that the algorithm works very well on small and medium scales, while on large scales the estimated trajectory drifts from the real one, as in the previous experiment.
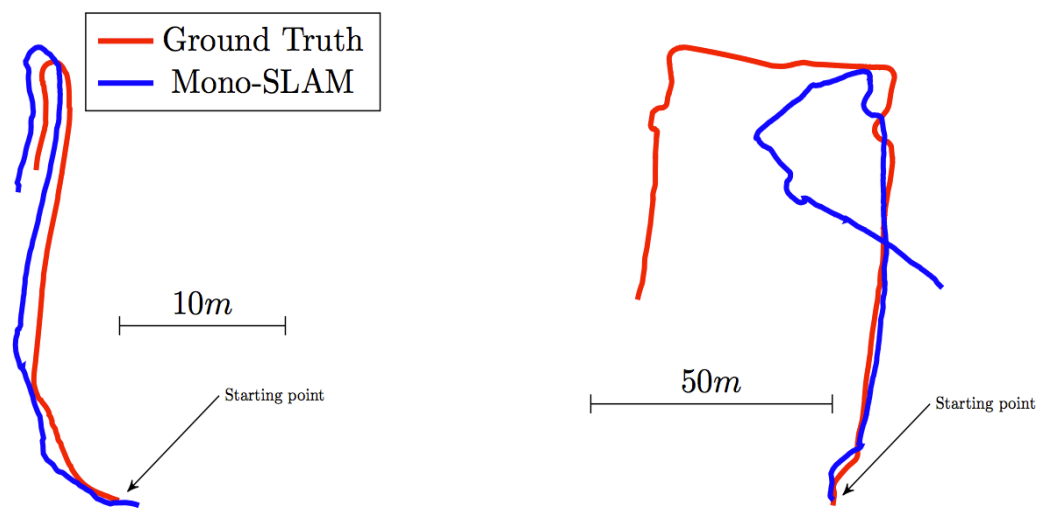

Figure 7. Results on the Bicocca 2009-02-26a dataset. Note that the small errors on estimated angles as well as scale drift both lead to a big difference in the final trajectories.
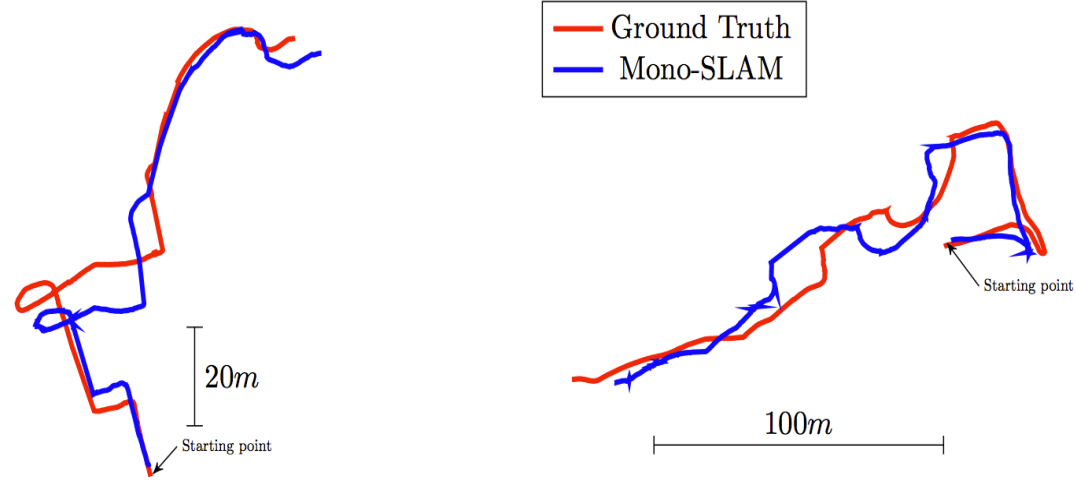

Figure 8: Results on the Bovisa 2008-10-04 dataset.

${ }^{3} \mathrm{http}: / /$ www.rawseeds.org/rs/datasets/view/7 


\section{CONCLUSIONS}

We presented a ROS implementation of the Mono-SLAM algorithm. The node is able to run in near real-time and is robust enough on small and medium scales to be used for retrieving the trajectory of the camera as well as re- constructing a 3D point-map of the environment. It should be noted that the performances of the algorithm are heavily influenced by the choice of parameters, in particular noise covariances. Moreover, some robustness issues still remain in the developed algorithm in the case of highly dynamic environments. Some solutions have been implemented in order to improve robustness, and actually the algorithm is able to work in dynamic environments and to correctly manage occlusions. Finally, when the camera trajectory is large, the computational time increases too much for meeting real-time constraints.

Future work will be devoted to improve the robustness of the approach on larger scales by implementing a way to detect and manage loop closings. Moreover, other sensors will be included when available, such as accelerometers, magnetometers, gyroscopes, and wheel odometry. Finally, more complex applications of the Mono-SLAM algorithm are under consideration. These applications concern a multi- robot extension of the algorithm; integration of high-level semantic informations in the algorithm; and an extension of the solution with multicamera systems.

\section{ACKNOWLEDGEMENTS}

The authors would like to thank everyone, just everyone!

\section{REFERENCES}

[1] Opencv library. Website. http://opencv.org.

[2] Radish: The robotics data set repository. Website. http://eigen.tuxfamily.org/.

[3] Ros (robot operating system). Website. http://www.ros.org.

[4] Josep Aulinas, Yvan Petillot, Joaquim Salvi, and Xavier Llado'. The slam problem: a survey. In Proceedings of the 2008 conference on Artificial Intelligence Research and Development: Proceedings of the 11th International Conference of the Catalan Association for Artificial Intelligence, pages 363-371, Amsterdam, The Netherlands, The Netherlands, 2008. IOS Press.

[5] Andrea Bonarini, Wolfram Burgard, Giulio Fontana, Matteo Mat- teucci, Domenico Giorgio Sorrenti, and Juan Domingo Tardos. Rawseeds: Robotics advancement through web-publishing of sensorial and elaborated extensive data sets. In In proceedings of IROS, volume 6, 2006.

[6] Simone Ceriani, Giulio Fontana, Alessandro Giusti, Daniele Marzo- rati, Matteo Matteucci, Davide Migliore, Davide Rizzi, Domenico G Sorrenti, and Pierluigi Taddei. Rawseeds ground truth collection systems for indoor self-localization and mapping. Autonomous Robots, 27(4):353-371, 2009.

[7] JavierCivera,AndrewDavison, andJMontiel.Dimensionlessmonoc- ular slam. Pattern Recognition and Image Analysis, pages 412-419, 2007.

[8] Javier Civera, Andrew J Davison, and J Montiel. Inverse depth parametrization for monocular slam. Robotics, IEEE Transactions on, 24(5):932-945, 2008.

[9]J avier Civera, Oscar G Grasa, Andrew J Davison, and JMM Montiel. 1-point ransac for extended kalman filtering: Application to real-time structure from motion and visual odometry. Journal of Field Robotics, 27(5):609-631, 2010.

[10] Javier Civera, Dorian Glvez-Lpez, Luis Riazuelo, Juan D. Tards, and J. M. M. Montiel. Towards semantic slam using a monocular camera. In IROS, pages 1277-1284. IEEE, 2011.

[11] Andrew J Davison. Active search for real-time vision. In Computer Vision, 2005. ICCV 2005. Tenth IEEE International Conference on, volume 1, pages 66-73. IEEE, 2005.

[12] Andrew J Davison, Ian D Reid, Nicholas D Molton, and Olivier Stasse. Monoslam: Real-time single camera slam. Pattern Analysis and Machine Intelligence, IEEE Transactions on, 29(6):1052-1067, 2007. 
[13] Ankur Handa, Margarita Chli, Hauke Strasdat, and Andrew J Davison. Scalable active matching. In Computer Vision and Pattern Recognition (CVPR), 2010 IEEE Conference on, pages 1546-1553. IEEE, 2010.

[14] Shahram Izadi, David Kim, Otmar Hilliges, David Molyneaux, Richard Newcombe, Pushmeet Kohli, Jamie Shotton, Steve Hodges, Dustin Freeman, Andrew Davison, et al. Kinectfusion: real-time 3d reconstruction and interaction using a moving depth camera. In Proceedings of the 24th annual ACM symposium on User interface software and technology, pages 559-568. ACM, 2011.

[15] Georg Klein and David Murray. Parallel tracking and mapping on a camera phone. In Mixed and Augmented Reality, 2009. ISMAR 2009. 8th IEEE International Symposium on, pages 83-86. IEEE, 2009.

[16] JMM Montiel, Javier Civera, and Andrew J Davison. Unified inverse depth parametrization for monocular slam. analysis, 9:1, 2006.

[17] Richard A Newcombe, Steven J Lovegrove, and Andrew J Davison. Dtam: Dense tracking and mapping in real-time. In Computer Vision (ICCV), 2011 IEEE International Conference on, pages 2320-2327. IEEE, 2011.

[18] Edward Rosten and Tom Drummond. Fusing points and lines for high performance tracking. In In International Conference on Computer Vision, pages 1508-1515. Springer, 2005.

[19] L. O. Russo, G. Airo` Farulla, M. Indaco, Rolfo D. Rosa, S., and B. Bona. Blurring prediction in monocular slam. International Design and Test Symposium, IEEE Conference, 2013.

[20] Renato F Salas-Moreno, Richard A Newcombe, Hauke Strasdat, Paul HJ Kelly, and Andrew J Davison. Slam++: Simultaneous localisation and mapping at the level of objects. Proc. Computer Vision and Pattern Recognition (CVPR), IEEE, June 2013.

[21] Hauke Strasdat, J. M. M. Montiel, and Andrew J. Davison. Editors choice article: Visual slam: Why filter? Image Vision Comput., 30(2):65-77, February 2012.

[22] Thomas Whelan, Michael Kaess, Maurice Fallon, Hordur Johannsson, John Leonard, and John McDonald. Kintinuous: Spatially extended kinectfusion. 2012. 